\title{
Hematoma subcapsular hepático pos-CPRE: reporte de caso y revisión de la literatura
}

\section{Subcapsular hepatic hematoma after ERCP. Case report and literature review}

Carlos Eduardo Rey-Chaves,,$^{1 *}$ Elkin Benítez, ${ }^{2}$ Fernando Gutíerrez, ${ }^{3}$ Juan Carlos Sabogal-Olarte. ${ }^{4}$

\author{
GaCCESO ABIERTO \\ Citación: \\ Rey-Chaves CE, Benítez E, Gutíerrez F, \\ Sabogal-Olarte JC. Hematoma subcapsular \\ hepático pos-CPRE: reporte de caso y revisión \\ de la literatura. Rev Colomb Gastroenterol. \\ 2021:36(Supl.1):59-62. https://doi. \\ org/10.22516/25007440.575 \\ Médico general, Hospital Universitario Mayor \\ Méderi. Bogotá, Colombia. \\ 2 Cirujano general, Universidad del Rosario. \\ Hospital Universitario Mayor Méderi. Bogotá, \\ Colombia. \\ 3 Residente de cirugía general, Universidad del \\ Rosario. Hospital Universitario Mayor Méderi. \\ Bogotá, Colombia. \\ ${ }^{4}$ Cirujano de hígado, vía biliar y páncreas, \\ Hospital Universitario Mayor Méderi. Bogotá, \\ Colombia. \\ *Correspondencia: Carlos Rey. \\ carlosrey991@gmail.com \\ Fecha recibido: $23 / 05 / 20$ \\ Fecha aceptado: $12 / 11 / 20$
}

\begin{abstract}
Resumen
Presentamos el caso de un paciente de 68 años, sin antecedentes de importancia, que por su cuadro clínico y resultados paraclínicos fue clasificado con riesgo intermedio para coledocolitiasis. En efecto, por medio de colangiorresonancia se realizó el diagnóstico confirmatorio. Fue llevado a colangiopancreatografía retrograda endoscópica en dos ocasiones -la primera fallida por papila intradiverticular-; en el segundo intento (exitoso con extracción del lito), el paciente presentó evolución posprocedimiento tórpida, con marcado dolor abdominal y anemización. Fue llevado a tomografía de abdomen con contraste, la cual confirmó el diagnóstico de hematoma subcapsular. Se decidió implementar el manejo conservador y se logró un control adecuado del sangrado.
\end{abstract}

\section{Palabras clave}

Colangiopancreatografía retrograda endoscópica, complicación, hematoma.

\section{Abstract}

This is the case of a 68-year-old female patient, with no relevant history, who was classified as intermediate risk for choledocholithiasis due to her symptoms and lab test results. The diagnosis was confirmed by means of cholangioresonance. She was taken to ERCP on two occasions; the first failed due to intradiverticular papilla, and during the second attempt (successful with the removal of the stone), the patient had a torpid postprocedure evolution, with marked abdominal pain and anemization. An abdominal tomography with contrast was performed, which confirmed the diagnosis of subcapsular hematoma. A conservative management was implemented, achieving adequate bleeding control.

\section{Keywords}

Endoscopic retrograde cholangiopancreatography; Complication; Hematoma.

\section{INTRODUCCIÓN}

La colangiopancreatografía retrógrada endoscópica (CPRE) es un procedimiento mínimamente invasivo que se utiliza como método terapéutico para diferentes patologías biliares o pancreáticas. Se creó en 1968 para visualizar el árbol biliar y desde 1974 fue posible realizar abordajes a la ampolla de Vater, como la papilotomía (1-3). Sin embargo, no es un procedimiento inocuo, presenta complicaciones en el 2,5\% a $8 \%$ de los casos, con mortalidad variable del $0,5 \%-1 \%$ (1, $3)$. Dentro de estas se resaltan en orden de incidencia la pancreatitis aguda ( $1 \%$ a $7 \%$ ), la bacteriemia ( $1,4 \%)$, el sangrado gastrointestinal-biliar (1\%), la perforación intestinal (1\%) y la colecistitis aguda $(0,2 \%)(1,3)$. Si bien la mortalidad por estas complicaciones no es muy alta $-0,5 \%-1 \%$ hasta $3 \%$ en algunas series de casos- $(1,3)$, la morbilidad de los pacientes 
es elevada y está representada por una estancia hospitalaria prolongada, requerimiento de estancia en unidad de cuidados intensivos (UCI), entre otras.

El hematoma subcapsular hepático posterior a CPRE corresponde a una complicación poco frecuente que puede tener desenlace fatal. La mayoría de la bibliografía disponible a nivel mundial consiste en reportes de caso.

\section{REPORTE DE CASO}

Paciente de 68 años, sin antecedentes de importancia, que ingresa al servicio de urgencias por cuadro clínico de 1 día de evolución consistente en dolor abdominal localizado en el hipocondrio derecho de tipo cólico, de alta intensidad, sin otra sintomatología asociada. Al examen físico se observa dolor abdominal en el hipocondrio derecho a la palpación, sin signos de irritación peritoneal.

En los resultados de laboratorio se evidencia alteración del perfil hepático (AST, ALT y fosfatasa alcalina elevadas) amilasa en rangos de normalidad, hemograma con leucocitosis y neutrofilia. Es llevado a ecografía hepatobiliar, donde se documenta colelitiasis sin colecistitis, barro biliar y prominencia de la vía biliar extrahepática.

Lo anterior clasifica al paciente con riesgo intermedio de coledocolitiasis, por lo que se realiza colangiorresonancia (CRNM), en donde se evidencia dilatación de la vía biliar intra y extrahepática secundaria a coledocolitiasis, con cálculo de $9 \mathrm{~mm}$ en el colédoco distal.

Por lo anterior, se decide realizar abordaje endoscópico por CPRE, el cual muestra papila intradiverticular alargada hasta la segunda porción del duodeno. Se intenta canular con guía y papilotomo hasta los conductos hepáticos; luego de 3 intentos e infundibulotomía con Needleknife, no es posible el ingreso a la vía biliar, por lo que se considera fallida. En un segundo intento de CPRE a los 5 días después del primer procedimiento se logra canalizar con guía metálica hasta los hepáticos; mediante papilotomía amplia sobre guía metálica se logra extracción de cálculo ovoide y grande del colédoco (Figura 1). Su evolución posprocedimiento fue satisfactoria hasta las 8 horas, cuando presenta súbitamente dolor abdominal, localizado en el hipocondrio derecho, de alta intensidad, sin signos de irritación peritoneal.

Ante el deterioro clínico, se solicita hemograma, el cual reporta anemia con una hemoglobina de $6,8 \mathrm{mg} / \mathrm{dL}$ y amilasa dentro de los límites normales. Con estos resultados y sin evidencia de sangrado digestivo activo, se decide solicitar tomografía axial computarizada (TAC) de abdomen con contraste, la cual confirma el diagnóstico de hematoma subcapsular hepático (Figura 2).

Luego de la evaluación clínica del paciente y ante su estabilidad hemodinámica, se inicia manejo conservador con repo-

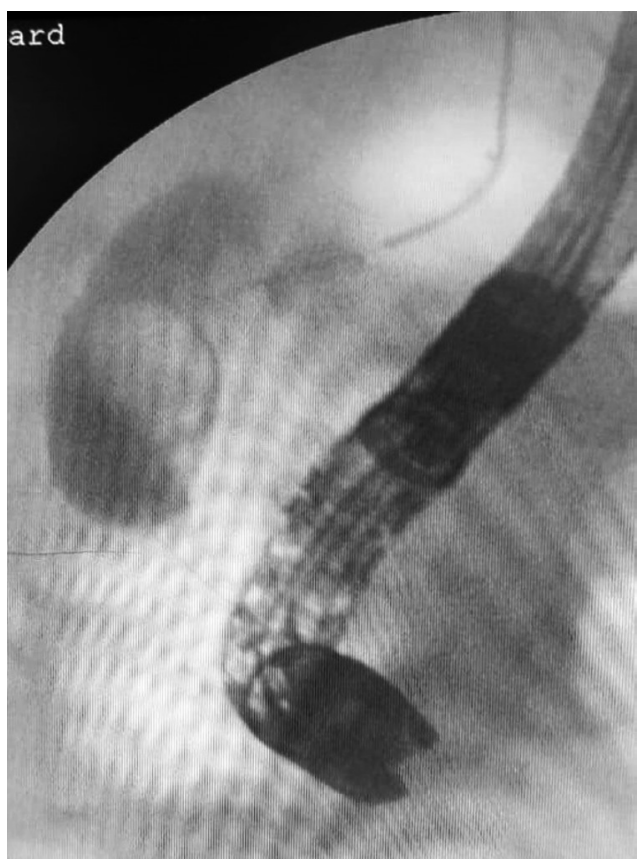

Figura 1. Fluoroscopia intraoperatoria-colangiopancreatografía retrógrada endoscópica, acceso a la vía biliar.

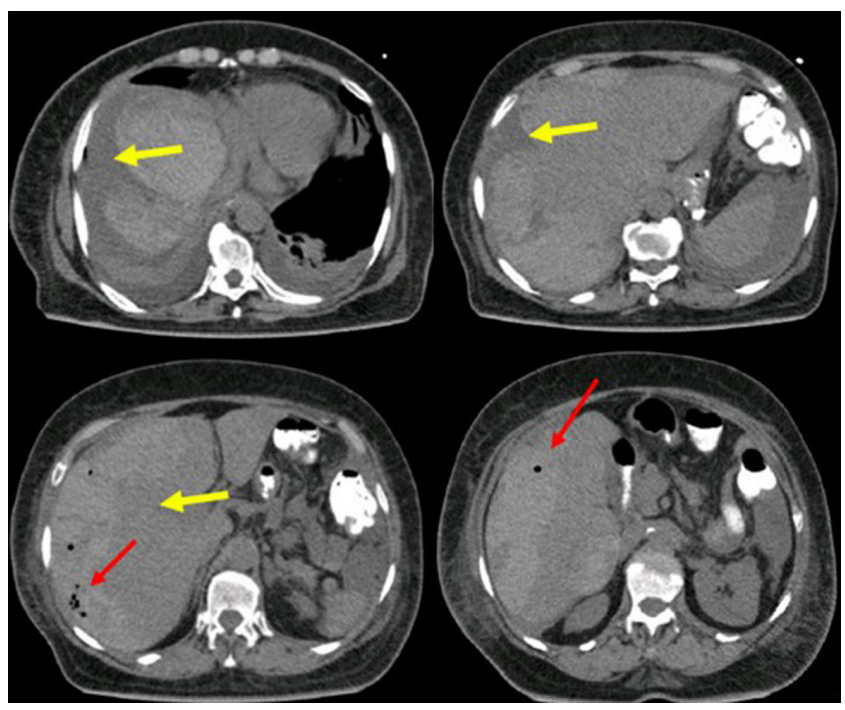

Figura 2. Tomografía axial computarizada de abdomen con contraste. Hematoma subcapsular hepático $206 \times 150 \times 70$; volumen: 1168 cc (flecha amarilla), con líquido libre en la cavidad y presencia de neumobilia (flecha roja).

sición hídrica, transfusión de 3 unidades de glóbulos rojos, inicio de antibiótico intravenoso y observación en UCI.

A las 72 horas del manejo instaurado, el paciente presenta una evolución satisfactoria, con control de su estado hemodinámico. Se solicita nueva tomografía de abdomen 
con contraste sin cambios con respecto a la imagen previa. Finalmente, con mejoría de la evolución clínica, se da egreso con antibiótico oral, analgesia y seguimiento ambulatorio por cirugía general y hepatobiliar.

\section{DISCUSIÓN}

El abordaje endoscópico de la vía biliar (colangiopancreatografía retrógrada endoscópica) es un procedimiento mínimamente invasivo, el cual, pese a que es seguro y presenta bajas tasas de mortalidad, reporta hasta un $7 \%$ de complicaciones $(2,4,5)$. En estas se contempla el sangrado gastrointestinal, el cual puede ser clínicamente significativo y que corresponde al $0,1 \%$ a $2 \%$ de los casos $(3,5)$, y aquellas que no presentan sangrado clínicamente significativo, que corresponden al $10 \%$ a $30 \%$ de los casos (5). Aunque la CPRE sea realizada por manos expertas, puede estar asociada a sangrado o hematoma que, de no ser diagnosticados oportunamente, pueden ser potencialmente mortales $(5,6)$.

El hematoma subcapsular hepático posterior a CPRE es una complicación rara, con bibliografía disponible consistente en reportes de caso $(1,5)$. En el 2001, Ortega y colaboradores publicaron el primer reporte de caso; desde entonces se han publicado casos similares (2).

De lo revisado en la literatura, la mayoría de los pacientes son mujeres, con una mediana de edad de 59 años; la gran mayoría de los pacientes presentan síntomas en las primeras 24 horas pos-CPRE, al igual que el caso descrito (7).

El dolor abdominal (91\%), posteriormente la anemia (43\%), la hipotensión (29\%) y la fiebre (20\%) son las formas de presentación más frecuentes; sin embargo, algunos pacientes son asintomáticos $(5,8)$. El examen diagnóstico de referencia es la tomografía computarizada, en donde se aprecia que el lóbulo hepático más afectado es el derecho en aproximadamente el $95 \%$ de los casos. Esta revisión sistemática muestra que el tratamiento médico es la mejor opción de manejo. La tasa de mortalidad reportada es del 9\% (8).

En cuanto a la causalidad, se describen múltiples teorías, como la punción accidental del árbol biliar con ruptura de los vasos del parénquima hepático o la fuerza excesiva en la tracción para la extracción de los cálculos (1-8).

Presentamos el caso del hematoma subcapsular más grande reportado hasta el momento, con unas dimensiones de $206 \times$ $150 \mathrm{~mm}$, que compromete ambos lóbulos hepáticos (el promedio en la literatura está entre $100 \times 130 \mathrm{~mm})(1-9)$.

El tratamiento depende de la evolución clínica, la estabilidad hemodinámica del paciente y los hallazgos imagenológicos (4). Se recomienda que el tratamiento se realice de forma multidisciplinaria (8).

Existen diferentes tipos de tratamiento, los cuales son: conservador, quirúrgico o por radiología intervencionista $(1,4)$.
El objetivo del manejo conservador (43,5\% de los casos) es la vigilancia estricta del estado hemodinámico $(1,5)$. Se indica en pacientes con estabilidad clínica cardiovascular y si el hematoma no comprime la vena hepática (4). Se recomienda el uso de antibióticos profilácticos por el riesgo de infección del hematoma, que se ha visto disminuye la mortalidad (8). Asimismo, se recomiendan los controles seriados de la hemoglobina y el control imagenológico $(3-5,8)$ (nivel de recomendación C).

El manejo por radiología intervencionista es una alternativa al tratamiento quirúrgico, que consiste en realizar embolización selectiva ( $26 \%$ de los casos) o superselectiva de los vasos o drenaje percutáneo del hematoma (17,4\% de los casos) (5) (nivel de recomendación C).

El manejo quirúrgico (13\% de los casos) se realiza únicamente cuando se presenta deterioro del estado general, inestabilidad hemodinámica, signos de irritación peritoneal, alto riesgo de ruptura del hematoma, líquido libre en la cavidad abdominal, extravasación del medio de contraste, falla del manejo conservador y si hay compresión extrínseca de la vena hepática $(1,4,8,9)$ (nivel de recomendación $\mathrm{C}$ ).

Si es necesario, también se hace seguimiento imagenológico para evaluar la progresión (5).

Dentro de la revisión de la literatura y de los casos reportados a nivel mundial, podemos definir que el hematoma subcapsular descrito anteriormente es el de mayor tamaño conocido hasta el momento. Es importante recalcar que el manejo conservador fue suficiente para el control de la complicación y que no requirió manejo intervencionista o quirúrgico. Lo anterior refleja la importancia de un manejo multidisciplinario y el impacto del manejo conservador pese al gran tamaño del hematoma, lo cual debe representar el estándar de manejo inicial en todos los casos.

\section{CONCLUSIONES}

El hematoma subcapsular pos-CPRE es una complicación poco frecuente del abordaje endoscópico de la vía biliar; sin embargo, de no ser diagnosticado a tiempo, puede ser potencialmente mortal. Dado el bajo número de casos a nivel mundial, se desconoce su incidencia verdadera. Sin embargo, es una complicación que debe estar presente durante el diagnóstico diferencial de aquellos pacientes cuya evolución pos-CPRE sea tórpida. El diagnóstico oportuno es la clave para evitar un desenlace fatal. Es importante recalcar que el presente caso es el de mayor tamaño reportado a nivel mundial; el manejo conservador fue exitoso y debe permanecer como el estándar de oro, aunque claramente ajustándolo a cada situación clínica de forma individual. 


\section{REFERENCIAS}

1. Orellana F, Irarrazaval J, Galindo J, Balbontin P, Manrquez L, Plass R, et al. Subcapsular hepatic hematoma post ERCP: A rare or an underdiagnosed complication? Endoscopy. 2012;44(S 02):E108-9. https://doi.org/10.1055/s-0031-1291493

2. García-Tamez A, López-Cossio A, Hernández-Hernández G, González-Huezo M, Rosales-Solís A, Corona-Esquivel E. Subcapsular hepatic hematoma: An unusual, but potentially life-threating post-ERCP complication. Case report and literature review. Endoscopia. 2016;28(2):75-80. https://doi.org/10.1016/j.endomx.2016.04.001

3. González-López R, García-Cano E, Espinosa-González O, Cruz-Salgado Á, Montiel-Jarquin ÁJ, Hernández-Zamora $\mathrm{V}$. Tratamiento quirúrgico para hematoma subcapsular hepático posterior a colangiografía retrógrada endoscópica; caso inusual. Cirugia y Cirujanos. 2015;83(6):506-9. https://doi.org/10.1016/j.circir.2015.05.028

4. Horn TL, Peña LR. Subcapsular hepatic hematoma after ERCP: case report and review. Gastrointest Endosc. 2004;59(4):594-6. https://doi.org/10.1016/s0016-5107(04)00013-6

5. Imperatore N, Micheletto G, Manes G, Redaelli DG, Reitano E, e Nucci G. Systematic review: Features, diagnosis, mana- gement and prognosis of hepatic hematoma, a rare complication of ERCP. Dig Liver Dis. 2018;50(10):997-1003. https://doi.org/10.1016/j.dld.2018.07.010

6. Koçak E, Ergül B, Akbal E, Köklü S, Karakayali AŞ. Subcapsular hepatic hematoma after ERCP. Acta GastroEnterol Belgica. 2012;67(2):379-80. https://doi.org/10.1016/j.gie.2007.06.008

7. Young Bang J, Coté GA. Rare and underappreciated complications of endoscopic retrograde cholangiopancreatography. Tech Gastrointest Endosc. 2014;16(4):195-201. https://doi.org/10.1016/j.tgie.2014.07.007

8. Zappa MA, Aiolfi A, Antonini I, Musolino CD, Porta A. Subcapsular hepatic haematoma of the right lobe following endoscopic retrograde cholangiopancreatography: Case report and literature review. World J Gastroenterol. 2016;22(17):4411-5. https://doi.org/10.3748/wjg.v22.i17.4411

9. Zizzo M, Lanaia A, Barbieri I, Zaghi C, Bonilauri S). Subcapsular Hepatic Hematoma After Endoscopic Retrograde Cholangiopancreatography A Case Report and Review of Literature. Medicine. 2015;94(26):pe1041. https://doi.org/10.1097/MD.0000000000001041 